\title{
Vesicular Monoamine and Glutamate Transporters Select Distinct Synaptic Vesicle Recycling Pathways
}

\author{
Bibiana Onoa, ${ }^{1,2}$ Haiyan Li, ${ }^{1,2}$ Johann A. Gagnon-Bartsch, ${ }^{5}$ Laura A. B. Elias, ${ }^{1,2,3}$ and Robert H. Edwards ${ }^{1,2,3,4}$ \\ Departments of ${ }^{1}$ Neurology and ${ }^{2}$ Physiology and Graduate Programs in ${ }^{3}$ Neuroscience and ${ }^{4}$ Cell Biology, University of California, San Francisco School of \\ Medicine, San Francisco, California 94158-2517, and 5Statistics Department, University of California at Berkeley, Berkeley, California 94720
}

Previous work has characterized the properties of neurotransmitter release at excitatory and inhibitory synapses, but we know remarkably little about the properties of monoamine release, because these neuromodulators do not generally produce a fast ionotropic response. Since dopamine and serotonin neurons can also release glutamate in vitro and in vivo, we have used the vesicular monoamine transporter VMAT2 and the vesicular glutamate transporter VGLUT1 to compare the localization and recycling of synaptic vesicles that store, respectively, monoamines and glutamate. First, VMAT2 segregates partially from VGLUT1 in the boutons of midbrain dopamine neurons, indicating the potential for distinct release sites. Second, endocytosis after stimulation is slower for VMAT2 than VGLUT1. During the stimulus, however, the endocytosis of VMAT2 (but not VGLUT1) accelerates dramatically in midbrain dopamine but not hippocampal neurons, indicating a novel, cell-specific mechanism to sustain high rates of release. On the other hand, we find that in both midbrain dopamine and hippocampal neurons, a substantially smaller proportion of VMAT2 than VGLUT1 is available for evoked release, and VMAT2 shows considerably more dispersion along the axon after exocytosis than VGLUT1. Even when expressed in the same neuron, the two vesicular transporters thus target to distinct populations of synaptic vesicles, presumably due to their selection of distinct recycling pathways.

\section{Introduction}

Monoamine neurotransmitters subserve a number of vital functions, including the control of movement, attention, motivation, emotional state, learning, and memory. Dopamine in particular has a central role in the neural pathway for reward (Wise, 2004). Environmental cues associated with reward produce the phasic release of dopamine from midbrain neurons by causing them to fire at high frequency, in bursts (Grace and Bunney, 1984b; Schultz, 2002). However, these cells also exhibit baseline pacemaker activity that contributes to tonic dopamine release (Grace and Bunney, 1984a). The ability of dopamine neurons to signal reward thus depends on their ability to increase extracellular dopamine more with burst firing than with pacemaking. Even though the increased dopamine overflow with burst firing reflects saturation of the dopamine transporter (Benoit-Marand et al., 2000), short-term synaptic depression due to synaptic vesicle depletion (Dittman et al., 2000) would reduce the amount of dopamine released by burst firing. Since most monoamines including dopamine do not activate ionotropic receptors, however, we know remarkably little about the properties of monoamine release.

Received Oct. 26, 2009; revised April 19, 2010; accepted April 27, 2010.

This work was supported by grants from National Institute on Drug Abuse and National Institute of Mental Health (NIMH) to R.H.E., and a diversity supplement from NIMH to B.O. We thank Sarah Elmes for her assistance with confocal microscopy, Doris Fortin for guidance in preparing neuronal cultures and live imaging, the Edwards laboratory for helpful discussion, and especially Prof. Terence Speed (Statistics Department, University of California at Berkeley) for his assistance with the statistical analysis.

Correspondence should be addressed to Robert H. Edwards, University of California, San Francisco School of Medicine, 600 16th Street, GH-N272B, San Francisco, CA 94158-2517. E-mail: robert.edwards@ucsf.edu.

DOI:10.1523/JNEUROSCI.5298-09.2010

Copyright $\odot 2010$ the authors $\quad 0270-6474 / 10 / 307917-11 \$ 15.00 / 0$
Electrochemical methods have been used to monitor dopamine release directly (Michael and Wightman, 1999; Mosharov et al., 2003). Voltammetric recording from striatal slices shows pronounced paired-pulse depression (Kennedy et al., 1992; Abeliovich et al., 2000), but release in vivo shows minimal depression despite high-frequency stimulation (Garris et al., 1994; Dugast et al., 1997; Benoit-Marand et al., 2001). However, electrochemistry measures dopamine overflow rather than synaptic release, and hence reflects diffusion and reuptake as well as the exocytosis (but not the endocytosis) of synaptic vesicles.

Reporters based on GFP can directly interrogate multiple aspects of the synaptic vesicle cycle. Fused to the lumenal domain of a synaptic vesicle membrane protein, the GFP variant superecliptic pHluorin shows almost complete fluorescence quenching at the low $\mathrm{pH}$ of synaptic vesicles. Its fluorescence thus increases dramatically with exposure to the extracellular medium during exocytosis, and decreases due to the rapid reacidification that follows endocytosis (Miesenböck et al., 1998; Sankaranarayanan et al., 2000). pHluorin fusions also monitor the trafficking of individual proteins, providing information complementary to other methods that monitor changes in cell membrane (Rizzoli and Betz, 2005).

Although synaptic vesicle proteins have generally been considered to recycle together, the analysis of pHluorin fusions has shown that they can differ substantially. After exocytosis, synaptic vesicle proteins mix to varying degrees with protein on the cell surface (Fernández-Alfonso et al., 2006; Voglmaier et al., 2006). Endocytosis can also vary as a function of neural activity and protein interactions (Ferguson et al., 2007). Indeed, a number of studies have suggested multiple pathways for synaptic vesicle re- 
cycling, including control of the fusion pore without full collapse into the plasma membrane ("kiss-and-run") and bulk endocytosis as well as clathrin-mediated endocytosis (Pyle et al., 2000; Gandhi and Stevens, 2003; Holt et al., 2003; Jockusch et al., 2005; Sara et al., 2005; Zhang et al., 2009). The electrochemical analysis of dopamine release from neurons in culture has in fact suggested the possibility of release by "kiss-and-run" (Staal et al., 2004), but we still know remarkably little about the synaptic vesicle cycle in monoamine neurons.

\section{Materials and Methods}

Molecular biology. The superecliptic pHluorin (a generous gift from J. Rothman, Yale University, New Haven, CT) was inserted between Gly-113 and Gly-114 of rat VMAT2, surrounded by different $5^{\prime}$ and $3^{\prime}$ linkers. The sequences of the $5^{\prime}$ linkers are GSGGTGSTSGGSGGTGGSGGTGG (long) and GSGGTGSTSGGSGGTGG (short), and the 3' linkers SGGTGGSGGTGGSGGTGGSGGTG (long) and SGGTGGSGGTGGSGGTG (short). The four pHluorin constructs were made with different combinations of linkers: $1,5^{\prime}$ long and $3^{\prime}$ long; $2,5^{\prime}$ short and $3^{\prime}$ short; $3,5^{\prime}$ long and $3^{\prime}$ short; and 4 (VMAT2-pHluorin), 5' short and $3^{\prime}$ long. To study dispersion, EGFP was fused to the N terminus of VMAT2 (Li et al., 2005) and VGLUT1. Colocalization of the two transporters was performed using a single plasmid that contains GFP-VGLUT1 followed by the internal ribosome entry site pIRES2 (BD), and HA-VMAT2 (Tan et al., 1998). cDNAs for transfecting COS-7 cells were subcloned into pcDNA3 (Invitrogen); and for transfecting neurons, into the pCAGGs vector that contains a modified chicken actin promoter (a generous gift from J. L. Rubenstein, University of California, San Francisco, San Francisco, CA).

Monoamine transport, tetrabenazine binding assays, and Western anal$y$ sis. COS-7 cells were transfected by electroporation, extracts prepared, and the uptake of ${ }^{3} \mathrm{H}$-serotonin assayed in triplicate at $29^{\circ} \mathrm{C}$ using $80 \mu \mathrm{g}$ of protein, as previously described (Finn and Edwards, 1997). To assay the binding of ${ }^{3} \mathrm{H}$-dihydrotetrabenazine, $80 \mu \mathrm{g}$ of protein was incubated for $30 \mathrm{~min}$ at $29^{\circ} \mathrm{C}$ in $200 \mu \mathrm{l}$ of $0.32 \mathrm{~m}$ sucrose $/ 10 \mathrm{~mm}$ HEPES-KOH, $\mathrm{pH}$ 7.4 ( $\mathrm{SH}$ buffer), and the reaction terminated by immediate dilution into $4 \mathrm{ml}$ of cold SH buffer containing $125 \mu \mathrm{M}$ unlabeled tetrabenazine, followed by filtration through $0.2 \mu \mathrm{m}$ HT 200 Tuffryn membranes (Pall). The filters were then dried and the bound radioactivity measured by scintillation counting in Cytoscint (ICN). Background binding for ${ }^{3} \mathrm{H}-$ dihydrotetrabenazine (TBZ) assay was determined using the same reaction solution with excess unlabeled tetrabenazine $(100 \mu \mathrm{M})$. Both the transport activity and TBZ binding experiments were performed in triplicate with 2-3 different membrane preparations from 2-3 independent transfections. For Western analysis, $10 \mu \mathrm{g}$ of protein from the transfected COS cell extracts was separated by electrophoresis through $10-20 \%$ polyacrylamide, transferred to Immobilon PVDF membrane (Millipore), and immunoblotted with a rabbit GFP antibody (Roche) followed by a secondary anti-rabbit antibody conjugated to HRP (GE Healthcare) and detected by enhanced chemiluminescence (Pierce Chemical).

Primary neuronal cultures. Hippocampal regions CA1-3 were dissected from $\mathrm{P} 0-\mathrm{P} 2$ rats, dissociated in $0.25 \%$ trypsin, washed in HBSS containing $10 \mathrm{~mm}$ HEPES and $20 \mathrm{~mm}$ glucose, triturated, and electroporated by Nucleofection with $0.25-0.4 \mu \mathrm{g}$ of DNA/2.5 $\times 10^{4}$ cells (Amaxa basic neuron SCN Nucleofector kit, Lonza Group). After transfection, the cells were allowed to recover for $10 \mathrm{~min}$, then plated in minimal essential medium containing $21 \mathrm{~mm}$ glucose, 5\% fetal bovine serum, $2 \%$ B27, 1\% GlutaMax, and Mito + serum extender (BD Biosciences) at a density of 700 cells $/ \mathrm{mm}^{2}$ onto coverslips coated with poly-L-lysine. Cytosine arabinoside at $3 \mu \mathrm{M}$ was added at 3-5 d in vitro (DIV) to inhibit glial growth, and neurons were refed with the same media containing cytosine arabinoside every $6 \mathrm{~d}$.

Postnatal rat midbrain cultures were prepared from the ventral mesencephalon of P0-P2 rats as previously described (Rayport et al., 1992; St-Gelais et al., 2004; Mani and Ryan, 2009), with several modifications. Briefly, the ventral midbrain (substantia nigra and ventral tegmental area) was digested in $20 \mathrm{U} / \mathrm{ml}$ papain (Worthington Biochemical) for 15 min at $35^{\circ} \mathrm{C}$ under humidified oxygen and mechanically dissociated, and the resulting cells were electroporated with $1-1.7 \mu \mathrm{g}$ of DNA/2-3 $\times 10^{6}$ cells. After recovery for $10 \mathrm{~min}$, the neurons were plated onto an astrocyte monolayer at a density of $1500-2000$ cells $/ \mathrm{mm}^{2}$. Two hours later, 10 $\mathrm{ng} / \mathrm{ml}$ glial-derived neurotrophic factor (Millipore), $6.7 \mu \mathrm{g} / \mathrm{ml} \mathrm{5-FU}$ (Sigma-Aldrich), and $16.5 \mu \mathrm{g} / \mathrm{ml}$ uridine were added to promote dopamine neuron survival and inhibit glia proliferation.

Immunocytochemistry. The dopaminergic identity of midbrain neurons was always confirmed by post hoc staining for tyrosine hydroxylase (TH). Transfected neurons grown for 12-25 DIV were fixed in PBS containing $4 \%$ paraformaldehyde, permeabilized, blocked, and stained in PBS containing $0.02 \%$ saponin, $1 \%$ fish gelatin, and $5 \%$ bovine serum albumin. Each coverslip was double stained using a rabbit antibody to TH and a chicken antibody to GFP (both Invitrogen), followed by a donkey anti-rabbit antibody conjugated to $\mathrm{Cy} 3$ or anti-chicken antibody conjugated to Cy2 (Jackson ImmunoResearch Laboratories).

To assess colocalization of the transfected transporters, both hippocampal and midbrain neurons were transfected as above with a single vector encoding both proteins, cultured for 15 DIV, fixed and permeabilized as above, then immunostained using rat anti-HA 3F10 (Roche) and chicken anti-GFP (Invitrogen). To restrict the comparison to presynaptic terminals, endogenous synaptophysin was also stained using a mouse monoclonal antibody (Sigma-Aldrich). In midbrain cultures, we further immunostained using rabbit anti-TH to ensure that the cells studied were dopaminergic. The primary antibodies were detected with donkey anti-rat conjugated to $\mathrm{Cy} 3$, anti-chicken conjugated to $\mathrm{Cy} 2$, and antimouse conjugated to Cy5 for hippocampal neurons; and with donkey anti-rat-Cy3, anti-chicken-Cy2, and anti-rabbit-Cy5 (Jackson ImmunoResearch Laboratories) and goat anti-mouse-Alexa 405 (Invitrogen) for midbrain neurons.

Confocal microscopy. For the analysis of transporter colocalization in fixed cells, stained puncta and/or neurites were identified under epifluorescence with a $100 \times / 1.3$ N.A. oil objective and then imaged by confocal laser microscopy using the following laser lines: $\sim 1.5 \mathrm{~mW}$ of the 488 $\mathrm{nm}$ Ar laser line for Cy2 (GFP-VGLUT1), $\sim 520 \mu \mathrm{W}$ of the $543 \mathrm{~nm} \mathrm{HeNe}$ laser line for Cy3 (HA-VMAT2), $\sim 1 \mathrm{~mW}$ of the $633 \mathrm{~nm}$ HeNe line for Cy5, and $93 \mathrm{~mW}$ of the $766 \mathrm{~nm}$ tunable Chameleon IR laser for Alexa 405. Emitted light was collected using a 500-550 bandpass filter, a 560 longpass filter, a 650-710 bandpass filter, and a 390-465 bandpass filter for Cy2, Cy3, Cy5, and Alexa 405 respectively. The pinhole was set at a mean field depth $\sim 205 \mu \mathrm{m}$ for Cy2, Cy3, and Cy5 and its maximum of $\sim 896$ $\mu \mathrm{m}$ for Alexa 405. The light was detected with two photomultiplier tubes at 8 bit sampling. The images are the mean of four continuous line scans at each wavelength.

Live imaging. For the live-cell imaging, cultures between 14 and 25 DIV were mounted in a laminar flow perfusion and stimulation chamber on an inverted Nikon TE300 fluorescence microscope, and images collected at $1 \mathrm{~Hz}$ under epifluorescence illumination using a xenon lamp with ET470/40 nm excitation and ET525/50 nm emission bandpass filters, with a $63 \times 1.2$ N.A. water objective, and a CCD camera (Hamamatsu ORCA ER) using $2 \times 2$ on-chip pixel binning. Action potentials (APs) were evoked by passing $1 \mathrm{~ms}$ bipolar current pulses through platinumiridium electrodes, to yield fields of $5-10 \mathrm{~V} / \mathrm{cm}$ at $10 \mathrm{~Hz}$. Except as otherwise noted, cells were continuously perfused at room temperature $\left(24^{\circ} \mathrm{C}\right)$ in standard Tyrode's solution (119 mm NaCl, $25 \mathrm{~mm}$ HEPES$\mathrm{NaOH}, \mathrm{pH} 7.4,2 \mathrm{~mm} \mathrm{CaCl}_{2}, 2 \mathrm{~mm} \mathrm{MgCl}_{2}, 2.5 \mathrm{~mm} \mathrm{KCl}$, and $30 \mathrm{~mm}$ glucose) containing $10 \mu \mathrm{M}$ 6-cyano-7-nitroquinoxaline-2,3-dione and $10 \mu \mathrm{M}$ 3-(2-carboxypiperazin-4-yl)propyl-1-phosphonic acid. Buffer containing $\mathrm{NH}_{4} \mathrm{Cl}$, pH 7.4, solution was prepared by substituting $50 \mathrm{~mm}$ $\mathrm{NaCl}$; acidic solutions, pH 5.5, were prepared by replacing HEPES with MES $\left(\mathrm{pK}_{\mathrm{a}}=6.1\right)$, and all other components were unchanged. To measure exocytosis alone, cells were preincubated with Tyrode's containing 0.6-1 $\mu \mathrm{M}$ bafilomycin A1 (baf) (Calbiochem) for 1 min before stimulation in the same solution. For the studies using FM4-64, the cultures were incubated in Tyrode's solution containing $15 \mu \mathrm{M}$ FM4-64 (Invitrogen), loaded by stimulation at $10 \mathrm{~Hz}$ for $60 \mathrm{~s}$, incubated in the same medium for an additional $60 \mathrm{~s}$ to allow internalization, and after washing for 10-15 min in medium without FM4-64, destained by stimulation at 10 $\mathrm{Hz}$ for $60 \mathrm{~s}$.

Data analysis and fitting. Fluorescence images were processed using MetaMorph 7.6 (MDS Analytical Technologies) and data analyzed using 
Wolfram Mathematica 7.0. In live cells, all the varicosities visible in $\mathrm{NH}_{4} \mathrm{Cl}$ were manually selected, the background subtracted, and the fluorescence intensity averaged over $4 \times 4$ pixel region of interest (ROI). For colocalization in fixed cells, boutons were selected by circling the perimeter of each fluorescent punctum and the integrated fluorescence computed. The fractional change in average fluorescence intensity normalized to the initial fluorescence $\left(\Delta F / F_{0}\right)$ of each ROI was plotted as a function of time, and when indicated in the text, renormalized to the maximum fluorescence after stimulation, or the total fluorescence in $\mathrm{NH}_{4} \mathrm{Cl}$. The averaged traces represent the fluorescence change at synaptic boutons collected from 5-7 coverslips in at least two independent transfections.

Transporter surface fraction and lumenal $\mathrm{pH}$ were calculated from the extent of quenching by Tyrode's solution containing MES buffer at $\mathrm{pH}$ 5.5 and the extent of unquenching in Tyrode's containing $50 \mathrm{~mm} \mathrm{NH}_{4} \mathrm{Cl}$, pH 7.4, as previously described (Mitchell and Ryan, 2004). The synaptic vesicle recycling fraction was calculated as the ratio of the steady-state $\Delta F / F_{0}$ reached after $600 \mathrm{APs}$ in the presence of baf to $\Delta F / F_{0}$ in $\mathrm{NH}_{4} \mathrm{Cl}$. The recycling fraction was binned in 0.1 intervals to plot the cumulative distribution.

Poststimulation endocytosis time constants were determined by fitting the poststimulus fluorescence decay in the absence of baf to a firstorder process: $F_{t}=B+e^{-t / a}(A-B)$, where $F_{t}=$ fluorescence at any given time $t, B=$ steady-state fluorescence after recovery, $A=$ surface fluorescence at the end of the stimulus and before recovery, and $a=$ $\tau_{\text {endo }}$, the time constant for poststimulus endocytosis. All traces that did not fit this model of exponential decay were eliminated from further analysis. The distribution of poststimulus $\tau_{\text {endo }}$ values fits well the sum of two Gaussians:

$$
P(a)=A_{1} \frac{e^{-\left(a-\mu_{1}^{2}\right) / 2 \sigma_{1}^{2}}}{\sqrt{2 \pi} \sigma_{1}}+A_{2} \frac{e^{-\left(a-\mu_{2}^{2}\right) / 2 \sigma_{2}^{2}}}{\sqrt{2 \pi} \sigma_{2}},
$$

where $A_{1}$ and $A_{2}$ are the areas under the curves. The goodness of the fit was verified by calculating the adjusted coefficient of determination $R_{\text {adjusted. The full width at half maximum (FWHM) was determined as }}^{2}$ FWHM $=2 \sqrt{2 \ln 2} \sigma$.

To calculate the rate of exocytosis during stimulation, we used a linear fit of the first $45 \mathrm{~s}$ of stimulation at $10 \mathrm{~Hz}$ in the presence of baf. Endocytosis traces during stimulation were obtained by subtracting the traces of the first $45 \mathrm{~s}$ in the absence of baf from those of the same cells in the presence of baf. When the resulting trace showed an initial delay followed by a linear increase, the endocytosis rate was determined by fitting the linear part of the response.

From time-lapse experiments using GFP-tagged transporters, the extent of dispersion $(D)$ was determined by averaging five $\Delta F / F_{0}$ values including the point at the end of the stimulus plus two points on each side. The dispersion amplitude $(D)$ distribution was binned in 0.05 intervals. The magnitude of reclustering $(R)$ was determined by averaging the last 30 values of $\Delta F / F_{0}$ in the plateau eventually reached. The fraction reclustered $\left(F_{\mathrm{r}}\right)$ was calculated as $F_{\mathrm{r}}=(R-D) / D$. The $F_{\mathrm{r}}$ distribution was binned in 0.1 intervals. The kinetic analysis of reclustering was restricted to those boutons where the recovery of fluorescence $\Delta F / F_{0}$ upon stimulation fit a first-order model $F_{t}=A-B\left(1-e^{-t / b}\right)$, where $F_{t}=$ fluorescence at any given time $t, A=$ fluorescence at the end of the stimulus and before recovery, $B=$ the difference from $A$ of the steady-state fluorescence after reclustering, and $b=\tau_{\text {reclustered }}$, the time constant for poststimulus reclustering.

The analysis of GFP-VGLUT1 and HA-VMAT2 colocalization was limited to puncta whose integrated fluorescence intensity equaled or exceeded $1000 \mathrm{U}$ after background subtraction. To quantify the colocalization per synapse, a colocalization index $(\gamma)$ was defined. This index relates the normalized intensities of the two fluorophores $A$ and $\mathrm{B}$ as follows: $\gamma=(B-A) /(B+A)$ with $A$ and $B=A^{\prime} / T_{\mathrm{A}}$ and $B^{\prime} / T_{\mathrm{B}}$, where $T_{\mathrm{A}}$ and $T_{\mathrm{B}}=$ sum of ROI fluorescence intensity for the two fluorophores and $A^{\prime}$ and $B^{\prime}=$ ROI intensities of each fluorophore. Thus, for $\gamma \approx 0.0$, the intensity ratio of both fluorophores is the same and they are completely colocalized; when $\gamma$ is negative, fluorophore A is more abundant; and when $\gamma$ is positive, fluorophore B predominates. The colocalization in- dex distribution was binned in 0.2 intervals. Colocalization was also characterized as the fluorescence intensity ratio $B^{\prime} / A^{\prime}$.

Statistical analysis. Two types of statistical test were performed: one to determine whether, for a given transporter, the data from different coverslips can be pooled; and the other to determine whether the observed differences in behavior of the two transporters are statistically significant.

For the first type of test, the null hypothesis is that, for a given transporter, measurements on different boutons are independent and identically distributed. Under this null hypothesis, there is no effect related to coverslip, and the data from different coverslips can be pooled. To test this possibility, we used a randomization test based on the F-statistic (Hooton, 1991; Mewhort, 2005). For each transporter (and each culture type), we calculated the standard $F$-statistic from the measurement of individual boutons grouped by coverslip, and denote this $F_{\mathrm{o}}$. The boutons were then randomly permuted among coverslips and a new $F$-statistic, $F^{*}$, was calculated; this was repeated 100,000 times. The estimated $p$ value is the fraction of $F^{*}$ values greater than or equal to $F_{\mathrm{o}}$. The majority of $p$ values were much $<0.05$, indicating that, in general, measurements from boutons on the same coverslip are not independent. We have therefore treated coverslips as the independent units of observation.

For the second type of test, the null hypothesis is that there is no difference in the distribution of measurements between coverslips expressing VMAT2- and VGLUT1-pHluorin. To test this null hypothesis, we first determined the median for each coverslip, then performed a permutation test on these medians, comparing VMAT2 to VGLUT1 (Hooton, 1991). In particular, for each transporter the mean of the medians was calculated; the test statistic was the absolute value of the difference of these two means:

$$
T_{0}=\operatorname{Abs}\left[\left(1 / n_{\mathrm{VMAT} 2}\right) \sum_{i} \operatorname{med}\left(c v_{i}^{\mathrm{VMAT} 2}\right)-\left(1 / n_{\mathrm{VGLUT} 1}\right) \sum_{i} \operatorname{med}\left(c v_{i}^{\mathrm{VGLUT1}}\right)\right],
$$

where $n_{\mathrm{VMAT} 2}$ and $n_{\mathrm{VGLUT} 1}$ are the number of coverslips for each transporter, $\mathrm{cv}_{i}^{\text {VMAT2 }}$ is the $i$ th VMAT2 coverslip, and $\mathrm{cv}_{i}^{\text {VGLUT1 }}$ is the $i$ th VGLUT1 coverslip. Next, we calculated 100,000 values of $T^{*}$ by randomly permuting the transporter labels and recalculating the test statistic. The estimated $p$ value is the fraction of $T^{\star}$ values greater than or equal to $T_{0}$. Note that because we look at the absolute value of the difference, the test is "two sided." Each estimated $p$ value is reported in both the corresponding results section and figure legend. The means reported in the text correspond to those obtained from Equation 2.

\section{Results \\ Localization of VMAT2 and VGLUT1 in transfected hippocampal and midbrain neurons}

Previous work has shown that both dopamine and serotonin neurons form glutamate synapses in culture (Johnson, 1994; Sulzer et al., 1998). The analysis of slice preparations has supported the corelease of glutamate by dopamine and serotonin neurons in vivo (Chuhma et al., 2004; Varga et al., 2009). Dopamine and serotonin neurons also appear to express low levels of vesicular glutamate transporter (VGLUT) mRNA in vivo as well as in vitro (Fremeau et al., 2002; Gras et al., 2002; Schäfer et al., 2002; Dal Bo et al., 2004; Mendez et al., 2008), although nondopamine midbrain neurons do as well (Yamaguchi et al., 2007). More recently, we have found that the synaptic vesicles of dopaminergic projections to the ventral striatum store glutamate as well as dopamine, and glutamate indeed promotes vesicle filling with dopamine (Hnasko et al., 2010). However, the extent of corelease has remained unclear, and previous work has even suggested the release of dopamine and glutamate from distinct processes (Hattori et al., 1991; Sulzer et al., 1998). We therefore compared the subcellular distribution of neuronal vesicular monoamine transporter VMAT2 with that of VGLUT1 as a representative member of the VGLUT family that has already been characterized (Voglmaier et al., 2006; Balaji and Ryan, 2007). 
To study the properties of VMAT2, we used postnatal cultures from the midbrain (Rayport et al., 1992). Although these cultures contain predominantly dopamine neurons, we immunostained them post hoc for the biosynthetic enzyme tyrosine hydroxylase to confirm the identity of imaged neurons. To determine whether any differences in behavior from VGLUT1 reflect differences in the transporter studied or the cellular environment in which it is expressed, we used postnatal hippocampal cultures, which contain predominantly glutamate neurons. In addition, to ensure expression of both transporters in the same cell, we produced a single construct that encodes a fusion of GFP to the $\mathrm{N}$ terminus of VGLUT1, followed by HAtagged VMAT2 under the independent translational control of an internal ribosome entry site (IRES). Since VMAT2 generally expresses at higher levels than VGLUT1, we attempted to equalize expression of the two proteins by inserting VMAT2 downstream of the IRES, which usually reduces expression.

After transfection into postnatal cultures of dissociated hippocampal neurons (Voglmaier et al., 2006), we observed a high degree of colocalization for VMAT2 and VGLUT1 at presynaptic sites identified by immunoreactivity for endogenous synaptophysin (Li et al., 2005) (Fig. 1A). A histogram of the colocalization index (described in Materials and Methods) shows a single peak, with no suggestion of distinct populations for VMAT2 or VGLUT1 alone (Fig. $1 B$ ).

In midbrain dopamine neurons, we find that VMAT2 and VGLUT1 usually but not always colocalize at presynaptic sites (Fig. 1A). In contrast to hippocampal cultures, midbrain dopamine neurons exhibit distinct populations of boutons expressing either VMAT2 alone or VGLUT1 alone, in addition to a large number of boutons where they colocalize (Fig. 1C). Midbrain dopamine neurons thus appear capable of segregating VMAT2 and VGLUT1 to distinct synaptic boutons.

\section{Development of an optical reporter to study the recycling of VMAT2}

To assess the recycling of VMAT2 at the nerve terminal in live cells, we developed an optical reporter for VMAT2. Since the N and $\mathrm{C}$ termini of VMAT2 are located in the cytoplasm, the ecliptic pHluorin was inserted into the first lumenal loop (Fig. 2A) using several linkers in multiple combinations (supplemental Fig. S1, available at www.jneurosci.org as supplemental material). Of four constructs produced, only three bound to the inhibitor tetrabenazine and transported ${ }^{3} \mathrm{H}$-serotonin, and of these, one could not be recognized by an antibody to GFP, suggesting defective folding of the pHluorin (Fig. 2 B, C; supplemental Fig. S1, available at www.jneurosci.org as supplemental material). Since it showed the higher transport activity, we chose one of the two remaining constructs (\#4 in supplemental Fig. S1, available at www.jneurosci.org as supplemental material) for all subsequent experiments. Transfected into primary hippocampal neurons, this VMAT2-pHluorin does not affect the uptake or destaining of styryl dye FM4-64 relative to untransfected cells in the same culture (Fig. 2D), suggesting that its expression does not interfere with the exocytosis or recycling of synaptic vesicles.

Expressed in hippocampal neurons, VMAT2-pHluorin exhibits many properties similar to VGLUT1. Like VGLUT1 (Voglmaier et al., 2006), VMAT2 shows little baseline fluorescence in physiological solution (Fig. $2 E-G$ ). Consistent with low cell surface expression, reducing external $\mathrm{pH}$ to 5.5 with the buffer MES quenches the fluorescence only slightly. Field stimulation at $10 \mathrm{~Hz}$ for $60 \mathrm{~s}$ causes a large, graded increase in fluorescence, strongly suggesting expression of VMAT2-pHluorin on synaptic vesicles (Fig. $2 E, F$ ). Indeed, the extent of stimulation for VMAT2 appears similar to VGLUT1. In addition, alkalinization of intracellular compartments with $\mathrm{NH}_{4} \mathrm{Cl}$ reveals higher levels of VMAT2 expression than VGLUT1 (Fig. 2E,F) despite using the 
A

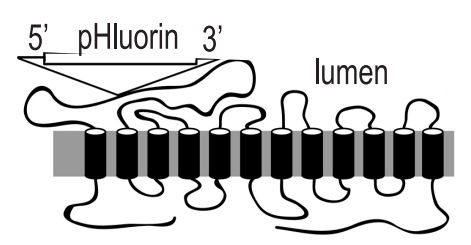

D

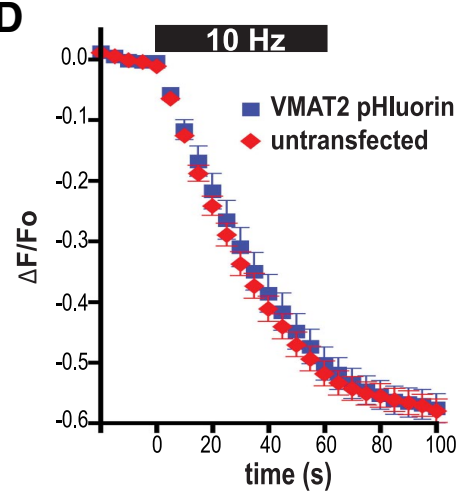

F

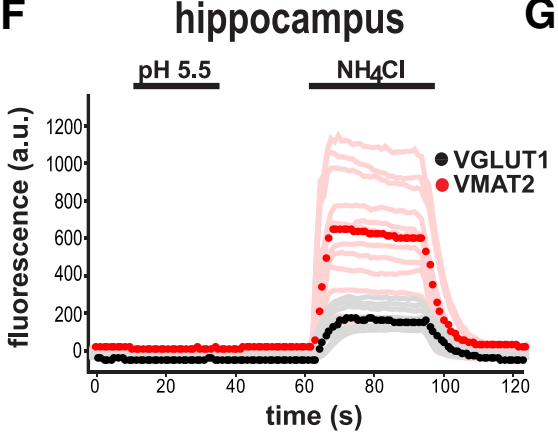

B

G
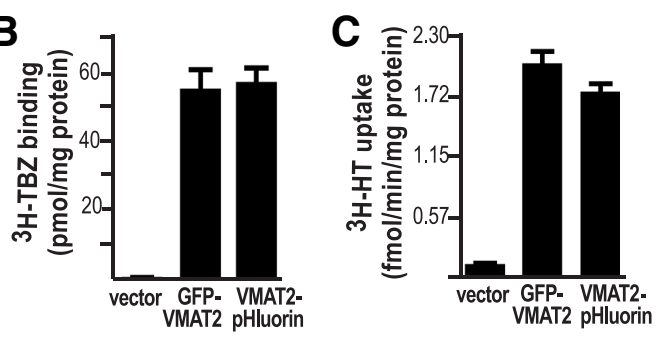

E rest MES
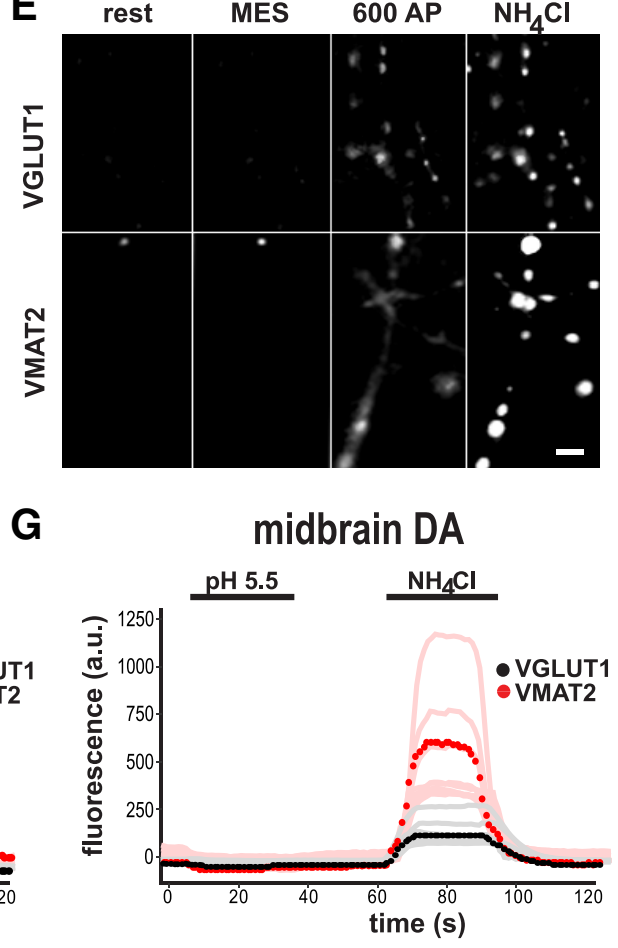

Figure 2. Development of a pHluorin-based reporter for VMAT2. $\boldsymbol{A}$, The superecliptic pHluorin was inserted into the first lumenal loop of VMAT2 using a combination of different linkers (supplemental Fig. $1 A$, available at www.jneurosci.org as supplemental material). The resulting cDNAs were transfected into COS cells and the extracts tested for expression by Western analysis, for binding to the noncompetitive inhibitor TBZ, and for the uptake of ${ }^{3} \mathrm{H}$-serotonin. One of the two constructs that met these criteria (VMAT2-pHluorin) was then compared directly to a fusion of GFP to the N terminus of VMAT2 (GFP-VMAT2) in terms of TBZ binding $(\boldsymbol{B})$ and serotonin uptake (C). D, Transfected into primary hippocampal culture, VMAT2-pHluorin does not affect the destaining of preloaded FM4- 64 by $10 \mathrm{~Hz}$ stimulation for 60 s. $E$, Hippocampal neurons transfected with either VGLUT1- or VMAT2-pHluorin were imaged at baseline (rest), upon acid quenching in MES buffer, $\mathrm{pH} 5.5$ for $20 \mathrm{~s}$, at the end of stimulation with 600 action potentials (600 AP), and upon total alkalinization for 20 s in $50 \mathrm{~mm} \mathrm{NH}_{4} \mathrm{Cl}, \mathrm{pH} 7.4$. F, Bouton fluorescence in hippocampal neurons transfected with VMAT2 ( $n=12$ coverslips from 3 cultures containing a total of 418 boutons) or VGLUT1 ( $n=8$ coverslips from 3 cultures containing 194 boutons) shows very low surface expression of the two transporters, but substantially higher total expression of VMAT2 than VGLUT1 after alkalinization with $\mathrm{NH}_{4} \mathrm{Cl}$. $\mathbf{G}$, The surface and total expression of VGLUT1 ( $n=6$ coverslips from 4 cultures containing a total of 253 boutons) and VMAT2 ( $n=8$ coverslips for 3 cultures containing 274 boutons) exhibited similar behavior in transfected midbrain dopamine neurons. Light traces in $\boldsymbol{F}$ and $\boldsymbol{G}$ indicate the coverslip means, and dark traces the means of the light traces.

same amount of cDNA for transfection, and all these properties of VMAT2-pHluorin were also observed in midbrain dopamine neurons (Fig. $2 G$ ). Further, in all of these experiments, the relative amounts of VMAT2- and VGLUT1-pHluorin expression were very similar in dopamine and hippocampal neurons.

The extent of fluorescence quenching in $\mathrm{pH} 5.5$ and of alkalinization in $\mathrm{NH}_{4} \mathrm{Cl}$ can be used to calculate the surface fraction of VMAT2-pHluorin and the $\mathrm{pH}$ of intracellular membranes where it resides (Mitchell and Ryan, 2004). The VMAT2 surface fraction (range 2.6-3.6\%) is similar to that of VGLUT1 (range $1.2-1.5 \%)$ in both hippocampal and midbrain dopamine neurons $(p=0.1$ for hippocampal and $p=0.6$ for dopamine neu- rons). In addition, the average $\mathrm{pH}$ of vesicles expressing VGLUT1 ( 5.8) does not differ significantly from those expressing VMAT2, in both cell populations ( $p=0.5$ for hippocampal and dopamine neurons).

To determine whether the properties observed might reflect excessive expression of the transporters and spillover onto compartments such as the plasma membrane where they would not normally reside, we examined cell surface expression as a function of total transport protein. Supplemental Figure S2 (available at www.jneurosci.org as supplemental material) shows very little correlation between total expression and cell surface fraction in both hippocampal and midbrain dopamine neurons, arguing against excessive expression.

\section{Accelerated endocytosis of VMAT2 during stimulation in midbrain dopamine neurons}

After stimulation, the decline in fluorescence of pHluorin-based reporters reflects the acidification that rapidly follows endocytosis (Sankaranarayanan and Ryan, 2000). Figure $3, A$ and $D$, shows that the poststimulus fluorescence decay or endocytosis (time constant, $\tau$, from first-order fitting kinetics) of VMAT2-pHluorin is substantially slower than that of VGLUT1pHluorin both in hippocampal neurons $(p=0.01)$ and in midbrain dopamine neurons $(p=0.06$; the null hypothesis was rejected, although the value is slightly higher than the conventional 5\% significance level). In addition, a histogram of the time constants for poststimulus endocytosis can be fitted by a bimodal distribution for both VGLUT1 and VMAT2 (Fig. $3 B, E$, Table 1 ), which may reflect expression of the reporters by different neuronal populations within these cultures. Surprisingly, the endocytosis of VMAT2pHluorin generally appears slower in dopamine than hippocampal neurons. Although the first VMAT2 peak (P1) narrows and becomes faster, the second peak (P2) broadens and becomes slower. Since a larger fraction of puncta expressing VMAT2-pHluorin did not fit our model even with slower kinetics, and were eliminated from consideration ( $~ 15 \%$ of boutons), the analysis may underestimate the difference between VMAT2 and VGLUT1.

To examine the endocytosis that occurs during stimulation, we evoked release in the presence and absence of the $\mathrm{H}^{+}$pump inhibitor bafilomycin. Bafilomycin blocks the fluorescence quenching that follows endocytosis, revealing only exocytosis. Subtraction from this value of the fluorescence increase observed in the absence of bafilomycin can thus be used to determine endocytosis during the stimulus (Sankaranarayanan and Ryan, 2001). In hippocampal neurons during stimulation, 
A hippocampus

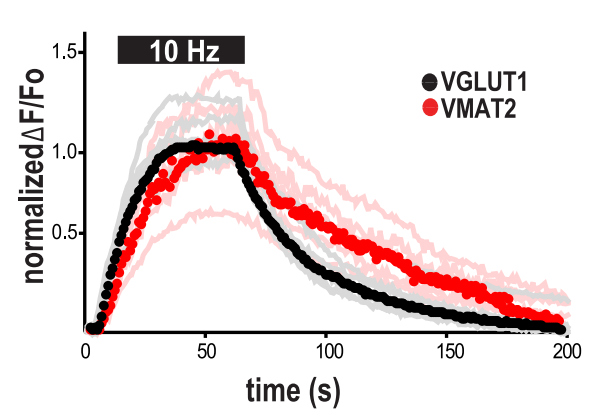

D midbrain DA

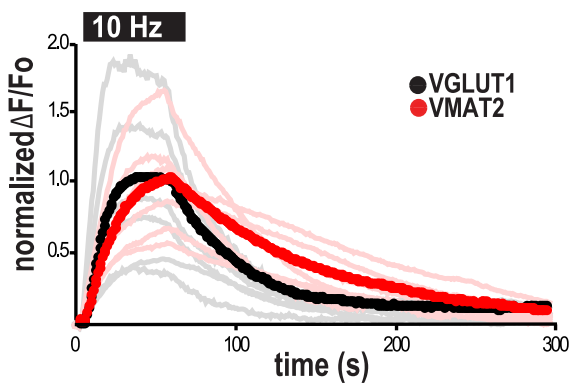

B

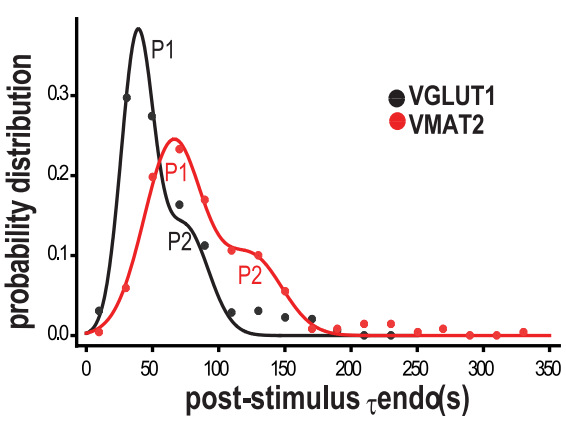

$\mathbf{E}$

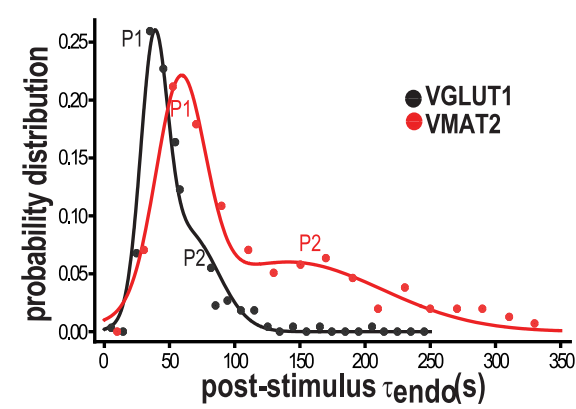

C

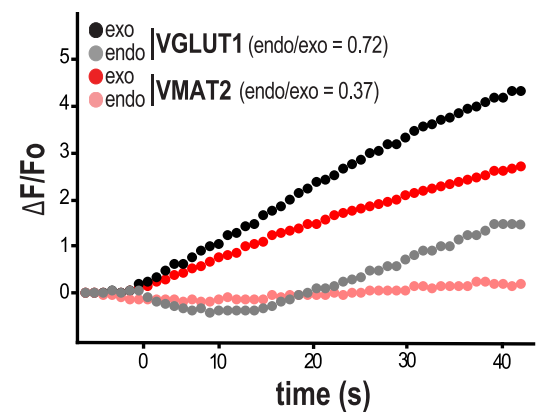

$\mathbf{F}$

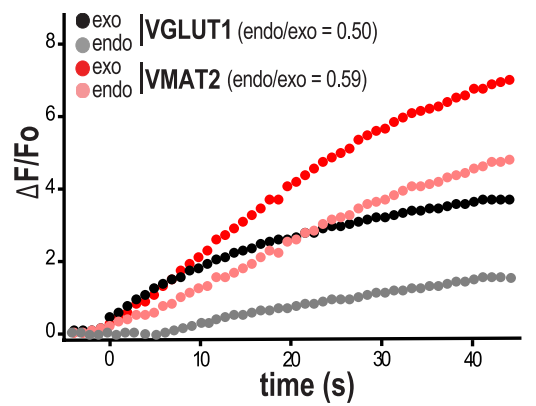

Figure 3. The kinetics of endocytosis differ between VMAT2 and VGLUT1 in both hippocampal and midbrain dopamine neurons. A, Time course of VGLUT1-pHluorin and VMAT2pHluorin fluorescence changes produced by $10 \mathrm{~Hz}$ stimulation for $60 \mathrm{~s}$ in hippocampal neurons, normalized to the maximum fluorescence. Fitting the fluorescence decay to a single exponential, the mean poststimulus $\tau_{\text {endo }}$ in terminals expressing VMAT2 is larger than in those expressing VGLUT1 ( $p=0.015 ; n=5-6$ coverslips from 3 cultures containing a total of $218-353$ boutons). $\boldsymbol{B}$, A histogram of poststimulus $\tau_{\text {endo }}$ shows a bimodal distribution for both transfected reporters that is well fitted by the sum of two Gaussians ( $R_{\text {adjusted }}^{2}>0.92$ for both fittings), and indicates that the poststimulus endocytosis of VGLUT1 is faster and more tightly distributed than that of VMAT2 in hippocampal neurons. C, The kinetics of exocytosis and endocytosis during stimulation were determined in the presence and absence of bafilomycin A1 (baf) for both VGLUT1 and VMAT2 reporters. Although VMAT2 is more highly expressed, the exocytosis of VGLUT1 is faster ( $p=0.015 ; n=4-6$ coverslips from 2 cultures containing a total of $81-99$ boutons). Despite a lag of 15-20 s, the endocytosis of VGLUT1 is also faster than VMAT2 $(p=0.003)$. $\boldsymbol{D}$, The normalized response of VGLUT1 and VMAT2 to stimulation of transfected midbrain dopamine neurons resembles that observed in hippocampal neurons $(\boldsymbol{A})$, with faster endocytosis of VGLUT1 ( $p=0.06$ the null hypothesis was rejected; $n=7$ coverslips from 3-4 cultures containing a total of 207-269 boutons). $E$, In midbrain dopamine neurons, the distribution of poststimulus $\tau_{\text {endo }}$ shows that the endocytosis of VGLUT1 is faster than that of VMAT2. As in hippocampal neurons, $\tau_{\text {endo }}$ exhibits a bimodal distribution ( $R_{\text {adjusted }}^{2}>0.94$ for both fittings). $F$, During stimulation, VMAT2 in midbrain dopamine neurons ( $n=3$ coverslips containing 152 boutons from 2 cultures) exhibits more rapid endocytosis than VGLUT1 ( $n=5$ coverslips containing 134 boutons from 2 cultures) ( $p=0.05$ ), in contrast to the results in hippocampal neurons. Further, the initial lag in endocytosis observed in hippocampal neurons does not occur in midbrain neurons. Light traces in $\boldsymbol{A}$ and $\boldsymbol{D}$ indicate coverslip means, and dark traces the mean of light traces. In $\boldsymbol{C}$ and $\boldsymbol{F}$, each trace indicates the mean of coverslip means, with coverslip means omitted for clarity.

Table 1. Time constants for endocytosis of VGLUT1- and VMAT2-pHluorin after stimulation in hippocampal and midbrain dopamine neurons

\begin{tabular}{|c|c|c|c|c|c|c|c|c|}
\hline & \multicolumn{4}{|c|}{ Hippocampal neurons } & \multicolumn{4}{|c|}{ Midbrain neurons } \\
\hline & \multicolumn{2}{|c|}{ VGLUT1 } & \multicolumn{2}{|c|}{ VMAT2 } & \multicolumn{2}{|c|}{ VGLUT1 } & \multicolumn{2}{|c|}{ VMAT2 } \\
\hline & P1 & P2 & P1 & P2 & P1 & P2 & P1 & P2 \\
\hline$\tau_{\text {endo }}(\mathrm{s})$ & 38.4 & 74.3 & 65.7 & 125.1 & 37.9 & 63.8 & 58.5 & 141.2 \\
\hline FWHM & 28.8 & 41.9 & 52.0 & 54.1 & 24.0 & 54.5 & 44.2 & 168.7 \\
\hline Area & 11.2 & 6.1 & 13.4 & 5.6 & 5.5 & 4.9 & 8.9 & 10.9 \\
\hline Mean $\tau_{\text {endo }}(s)^{a}$ & 52.37 & & 81.3 & & 50.2 & & 100.8 & \\
\hline
\end{tabular}

P1, Peak1; P2, peak 2; mean $\tau_{\text {endo }}$ values are the means of the total number; ${ }^{a}$ Means were calculated as indicated in the Materials and Methods.

VGLUT1 again displays higher rates of endocytosis $\left(0.06 \mathrm{~s}^{-1}\right)$ than VMAT2 $\left(0.01 \mathrm{~s}^{-1}\right)(p=0.003)$ (Fig. $\left.3 C\right)$. Despite an initial 15-20 s delay, the endocytosis of VGLUT1 was almost able to compensate for exocytosis ( rate $_{\text {endo }}=0.72$ rate $_{\text {exo }}$ ). In monoamine neurons, however, the endocytosis of VMAT2 $\left(0.10 \mathrm{~s}^{-1}\right)$ is faster than that of VGLUT1 $\left(0.032 \mathrm{~s}^{-1}\right)(p=$ 0.05 ) (Fig. $3 F$ ), and the delay before endocytosis eliminated only for VMAT2. The recycling of VMAT2 and hence synaptic vesicles that can store monoamines is thus controlled by a combination of cell-specific trafficking machinery, VMAT2, and activity.
Differential targeting of VMAT2 and VGLUT1 to the synaptic vesicle recycling pool

We also used VMAT2-pHluorin to assess the exocytosis evoked by repeated stimulation. Despite the higher level of VMAT2pHluorin expressed (Fig. 2F, G), the initial rate of exocytosis is faster for VGLUT1 $\left(0.09 \mathrm{~s}^{-1}\right)$ than VMAT2 $\left(0.04 \mathrm{~s}^{-1}\right)$ in hippocampal neurons $(p=0.01)$, but faster for VMAT2 $\left(0.17 \mathrm{~s}^{-1}\right)$ than VGLUT1 $\left(0.06 \mathrm{~s}^{-1}\right)$ in dopamine neurons $(p=0.02)$ (Fig. $3 C, F)$, indicating both transporter- and cell-specific determinants of release. The total fluorescence increase $(\Delta F)$ produced by stimulation is also very similar for both reporters (supplemen- 
A
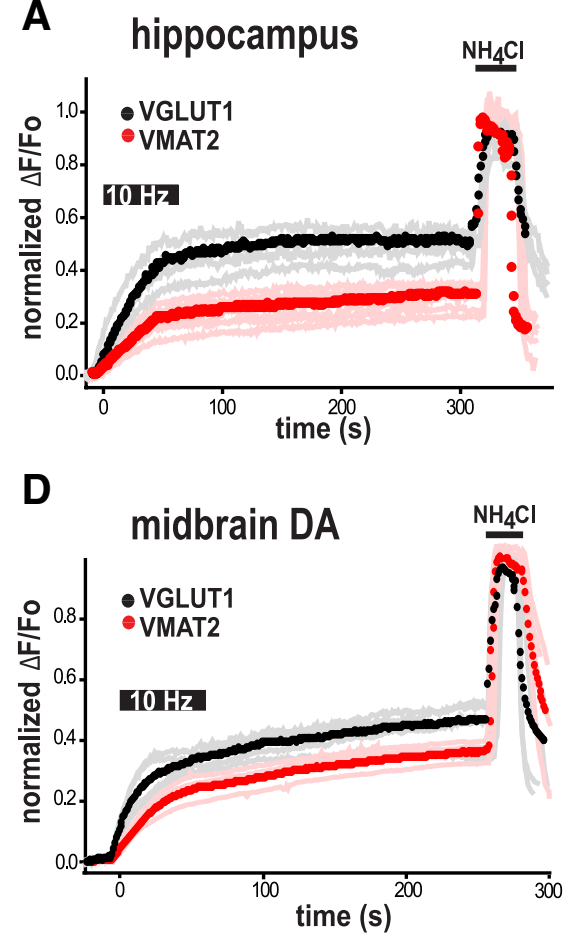

B

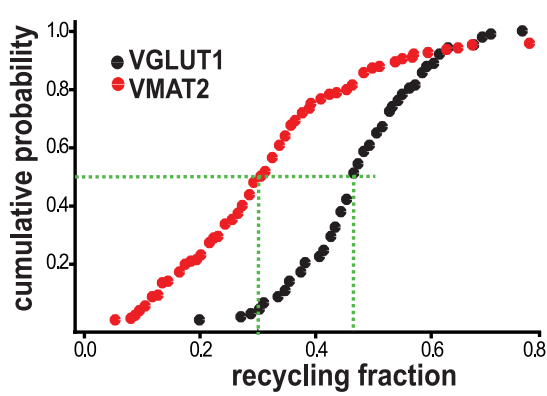

$\mathbf{E}$

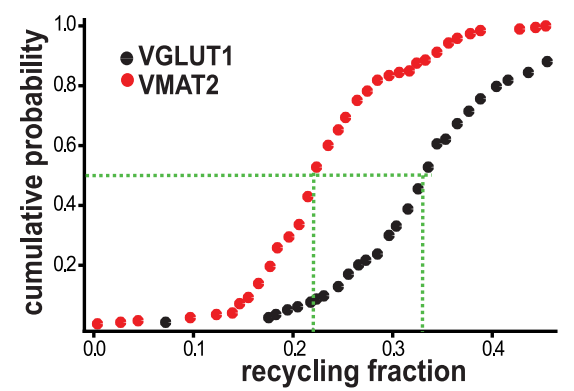

C

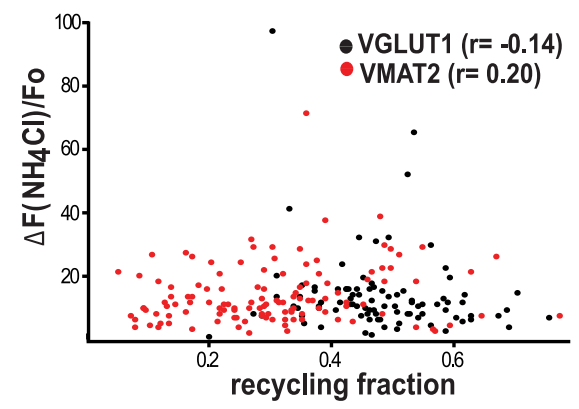

$\mathbf{F}$

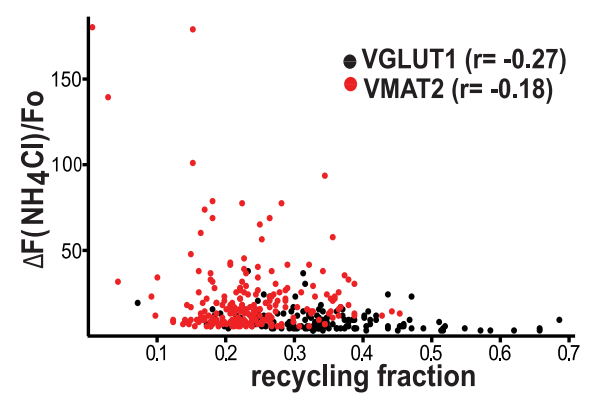

Figure 4. A smaller proportion of VMAT2 undergoes stimulated exocytosis than VGLUT1. A, Time course of fluorescence changes for hippocampal neurons transfected with VGLUT1 ( $n=4$ coverslips containing a total of 92 boutons from 2 cultures) or VMAT2-pHluorin cDNAs ( $n=6$ coverslips containing 125 boutons from 2 cultures) and stimulated at $10 \mathrm{~Hz}$ for $60 \mathrm{~s}$ in the presence of bafilomycin, normalized to total fluorescence in $\mathrm{NH}_{4} \mathrm{Cl}$. A substantially smaller fraction of VMAT2 undergoes exocytosis in response to stimulation than VGLUT1 ( $p=$ 0.005 ). $\boldsymbol{B}$, Cumulative probability distribution of the recycling fraction (ratio of maximal fluorescence at the end of stimulation to total fluorescence in $\mathrm{NH}_{4} \mathrm{Cl}$ ). A cumulative probability of $50 \%$ corresponds to a recycling fraction of 0.46 in the case of VGLUT1 and 0.30 in the case of VMAT2 (green dotted lines). $C$, The recycling fraction determined does not correlate with the expression of reporter (revealed in $\mathrm{NH}_{4} \mathrm{Cl}$ ). D, The analysis of midbrain dopamine neurons transfected with VGLUT1 ( $n=4$ coverslips containing 134 boutons from 2 cultures) or VMAT2 ( $n=5$ coverslips containing 226 boutons from 2 cultures) also shows a smaller recycling pool size for $\operatorname{VMAT2}(p=0.008)$, similar to hippocampal neurons. $\boldsymbol{E}$, A cumulative probability of $50 \%$ corresponds to a recycling fraction of 0.33 for VGLUT1 and 0.22 for VMAT2. $\boldsymbol{F}$, The recycling fraction does not correlate with reporter expression in midbrain dopamine neurons. In $\boldsymbol{A}$ and $\boldsymbol{D}$, light traces indicate coverslip means and dark traces the means of the light traces.

tal Fig. S3, available at www.jneurosci.org as supplemental material). Considering the differences in protein expression, VMAT2 and VGLUT1 must therefore differ in their targeting to synaptic vesicles capable of evoked release, i.e., the recycling pool capable of regulated exocytosis, as opposed to resting or reserve pool vesicles that are not available (Rizzoli and Betz, 2005; FernandezAlfonso and Ryan, 2008).

To assess the proportion of each transporter on recycling pool vesicles, we used prolonged stimulation in the presence of bafilomycin. Stimulation at $10 \mathrm{~Hz}$ for $60 \mathrm{~s}$ is generally considered sufficient to release most of the vesicles in the recycling pool (Fernandez-Alfonso and Ryan, 2008), and, consistent with this, we find that the fluorescence begins to plateau toward the end of the stimulus (Fig. 4A,D). Importantly, the difference in extent of exocytosis between VMAT2 and VGLUT1 persists even after stimulation with 1200 action potentials (supplemental Fig. S4, available at www.jneurosci.org as supplemental material). Normalization of the fluorescence produced by stimulation in the presence of bafilomycin to the fluorescence observed in $\mathrm{NH}_{4} \mathrm{Cl}$ can then be used to determine the proportion of pHluorin-tagged protein that resides in the recycling pool (recycling fraction). In hippocampal neurons, a substantially smaller proportion of VMAT2 (0.3) than VGLUT1 (0.47) is available for evoked release $(p=0.005)$, and the same holds true for midbrain dopamine neurons $(p=0.008)$ with a recycling fraction of 0.23 for VMAT2 and 0.3 for VGLUT1 (Fig. $4 A, D$ ). To eliminate any bias against boutons that do not respond to stimulation, we cotransfected cherry-synaptophysin, and selected boutons for analysis based solely on the expression of that reporter. The data acquired with cotransfection of cherry-synaptophysin showed no difference from those obtained without, and the results were therefore pooled. However, we did observe a substantial number of boutons with essentially no evoked exocytosis of VMAT2. A cumulative frequency histogram confirms this observation and shows that, in both cell populations, at least $50 \%$ more VGLUT1 is available for regulated release than VMAT2 (Fig. $4 B, E$ ). It is also important to note that the size of the recycling fraction does not correlate with the amount of reporter expressed (Fig. 4C,F), indicating that the differences observed do not reflect saturation of the sorting machinery.

In contrast to differences in endocytosis, differences in the extent of exocytosis can be used to infer expression on distinct populations of synaptic vesicles. The endocytosis of a membrane protein such as VMAT2 or VGLUT1 is a function of both the specific endocytic pathway used and the rate at which the protein targets to that pathway, so differences in the rate of endocytosis may only reflect differences in the rate of targeting to the same endocytic pathway. However, exposure of one protein at the cell surface without exposure of another means that they must reside on different membranes. The differences in extent of exocytosis thus indicate that a larger proportion of VMAT2 than VGLUT1 resides on the reserve pool of synaptic vesicles. 
VGLUT1 and VMAT2 differ in their activity-dependent dispersion and reclustering

In the course of these experiments, we observed substantially more long-range axonal movement of $\mathrm{VMAT}^{+}$than VGLUT1 ${ }^{+}$membranes, even in the absence of stimulation. Although this mobility reflects anterograde and retrograde movement of discrete axonal transport vesicles where the pHluorin remains unquenched, it suggests that the two proteins may also differ in their dispersion and reclustering after evoked release, differences that could account for the observed differences in endocytosis and recycling pool size. Both peripheral and integral membrane proteins of the synaptic vesicle generally move into the axonal plasma membrane with stimulation, then recluster after endocytosis (Chi et al., 2001; Li and Murthy, 2001; Fortin et al., 2005). This graded dispersion and reclustering differ from the directed movement of individual puncta along the axon observed even in the absence of stimulation, but imaging of the pHluorin reporters had also suggested greater activity-dependent movement of VMAT2 into the axon than VGLUT1 (Fig. 2E). Since the N terminus of both transporters resides in the cytoplasm, and is therefore insensitive to changes in lumenal $\mathrm{pH}$ that occur with exocytosis and endocytosis, we used $\mathrm{N}$-terminal GFP fusions to quantify the extent of dispersion and reclustering. Figure $5 \mathrm{~A}$ shows that VMAT2 undergoes a larger decline in fluorescence over the bouton (mean amplitude -0.31 ) and hence greater dispersion into the axon than VGLUT1 $(-0.44 ; p=0.02)$, even though the pHluorin reporters had indicated similar amounts of exocytosis. A histogram of the dispersion amplitudes confirms that this difference involves the full range of boutons, not simply a subset (Fig. 5B).

We also examined the rate and extent of fluorescence recovery at the bouton after stimulation. Only a minority of boutons exhibit full recovery in the time frame of these experiments ( $5 \mathrm{~min}$ ), but Figure $5 C$ shows that VGLUT1 fluorescence returns to at least some extent at most sites (average fraction reclustered $=0.4$ ). In contrast, VMAT2 shows no recovery at almost half of the boutons. Further, after excluding those sites where the return of fluorescence does not exhibit first-order kinetics (75\% of VMAT2 ${ }^{+}$ and $54 \%$ of VGLUT $1^{+}$boutons), we find that VMAT2 recovers more slowly than VGLUT1 at the remaining boutons ( $p=0.01$ ) (Fig. $5 D$ ). VMAT2 thus differs from VGLUT1 in both the extent of dispersion during and rate of recovery after stimulation.

\section{Discussion}

The results show that VMAT2 colocalizes with VGLUT1 at a large proportion of the boutons formed by hippocampal and midbrain dopamine neurons, consistent with recent work indicating a role for glutamate in vesicle filling with dopamine (Hnasko et al., 2010). However, in contrast to hippocampal neurons, where VMAT2 colocalizes with VGLUT1 at essentially all boutons,
B

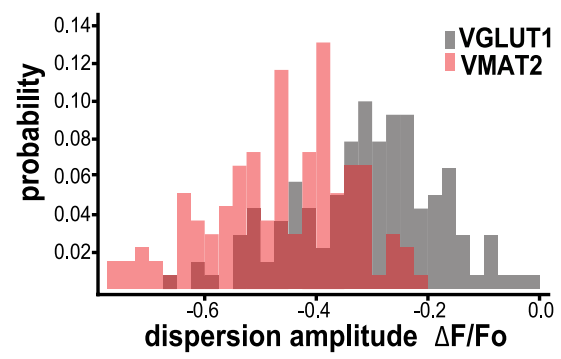

D

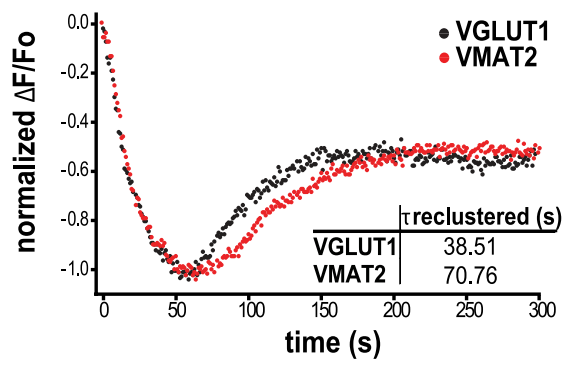

Figure 5. VMAT2 exhibits more stimulus-induced dispersion than VGLUT1. A, Time course of fluorescence changes for GFPVGLUT1 ( $n=5$ coverslips containing 141 boutons from 2 cultures) and GFP-VMAT2 ( $n=5$ coverslips containing 138 boutons from cultures) at the boutons of transfected hippocampal neurons stimulated at $10 \mathrm{~Hz}$ for $60 \mathrm{~s}$. Light traces indicate coverslip means,

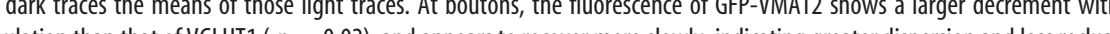
gextent of fluorescence recovery after stimulation shows that a large from selected boutons. $\boldsymbol{D}$, The kinetics of reclustering was calculated by fitting to a single exponential function the traces that show poted for those where recovery occurs (46\% of boutons for VGLUT1 and 25\% for VMAT2). Although the extent of reclustering in e kinetics are not $(p=0.01)$.

VMAT2 segregates from VGLUT1 in a substantial proportion $(\sim 27 \%)$ of the boutons made by dopamine neurons. The frequency of $\mathrm{VMAT}_{2}{ }^{+} / \mathrm{VGLUT}^{-}$boutons $(\sim 20 \%)$ exceeds that of VMAT2 ${ }^{-} / \mathrm{VGLUT1}^{+}(\sim 7 \%)$, suggesting that some of the former might represent somatodendritic sites. In that case, however, we might have expected a similar phenomenon in hippocampal neurons, which showed no segregation of the two transporters. Midbrain dopamine neurons may thus have the capacity for dopamine and glutamate release from distinct sites, in addition to corelease from the same vesicles. Indeed, this segregation is consistent with previous work in vivo suggesting that dopamine neurons form two distinct types of synapse, one en passant that presumably releases dopamine and the other an asymmetric synapse characteristic of glutamatergic transmission (Hattori et al., 1991).

To visualize the exocytosis and endocytosis of VMAT2 at individual boutons, we constructed VMAT2-pHluorin, which retains both drug binding and transport activity, and does not appear to perturb the cycling of synaptic vesicles as examined using the styryl dye FM4-64. Similar to VGLUT1-pHluorin, VMAT2-pHluorin exhibits a very small surface fraction at baseline, conferring a high ratio of signal to noise on stimulation. The vesicular $\mathrm{pH}$ in both types of neurons seems not to be affected by the presence of either transporter as previously reported for synaptopHluorin (Mani and Ryan, 2009).

Using VMAT2-pHluorin to characterize the synaptic vesicle cycle, we find that after stimulation, VMAT2 undergoes endocytosis more slowly than VGLUT1. This difference between the two 
transporters occurs in both hippocampal and midbrain dopamine neurons, indicating that it reflects the properties of the two proteins rather than the cells in which they are expressed. The distribution of time constants for poststimulus endocytosis further indicates two distinct types of synapse, perhaps due to expression of the reporters by distinct neuronal populations (GABAergic as well as glutamatergic and dopaminergic) within each of the culture systems. However, none of the endocytic time constants determined (38-140 s) are consistent with the rapid recycling ( $<1 \mathrm{~s}$ ) observed for "kiss-and-run" (Pyle et al., 2000; Gandhi and Stevens, 2003; Zhang et al., 2009), and may therefore correspond to bulk as well as clathrin-dependent endocytosis (Sun et al., 2002; Jockusch et al., 2005; Granseth et al., 2006; Xu et al., 2008).

In contrast to poststimulus endocytosis of both transporters, endocytosis of VMAT2 during the stimulus shows differences between hippocampal and midbrain dopamine neurons. During stimulation, the endocytosis of VMAT2 is faster in dopamine than glutamate neurons, whereas the endocytosis of VGLUT1 appears faster in hippocampal neurons. The VMAT2 reporter is in fact more highly expressed than VGLUT1-pHluorin, but the difference in expression appears similar in both cell populations, and thus cannot account for the observed cell-specific differences in internalization of VMAT2. Consistent with more efficient recycling, midbrain dopamine neurons use less of their recycling pool during high-frequency stimulation (Mani and Ryan, 2009), and the mechanism may involve either the acceleration of a preexisting endocytic pathway or the activation of a distinct pathway with faster kinetics.

The analysis of recycling pool size supports the differential targeting of VMAT2 and VGLUT1. In hippocampal neurons, the proportion of multiple synaptic vesicle proteins in the recycling pool approaches $\sim 50 \%$, and it has not previously been possible to identify factors that regulate the size of this pool (Fernandez-Alfonso and Ryan, 2008). In contrast, we now find that the proportion of VMAT2 in the recycling pool is substantially lower than that of VGLUT1, and this does not reflect increased expression of the VMAT2 reporter. Although the similar response to stimulation suggests that many synaptic vesicles contain both transporters, the ability to evoke release of more VGLUT1 than VMAT2 indicates that a larger proportion of VGLUT1 resides on the recycling pool, and conversely, that a larger proportion of VMAT2 resides on the reserve pool of vesicles, which does not respond in the same way to stimulation.

Recent work using FM dyes has shown that dopamine neurons have a smaller recycling pool size (and hence lower probability of release) than hippocampal neurons (Daniel et al., 2009). However, FM dyes label the membrane of all recycled synaptic vesicles, not simply those that express VMAT2 or VGLUT1. Since a larger proportion of VGLUT1 resides in the recycling pool, we might expect VGLUT1 to exhibit a smaller recycling pool size in dopamine neurons, and Figure 4, $B$ and $E$, supports this possibility. On the other hand, VMAT2 recycling pool size shows little difference between the two populations. Further, the difference between VMAT2 and VGLUT1 expressed in the same neuronal population indicates that these differences in recycling pool size reflect differences in the transporter, as well as the cell type. Although the time constant of FM dye destaining does not appear to differ between dopamine and hippocampal neurons (Daniel et al., 2009), we also find differences in the relative rates of evoked exocytosis for VMAT2 and VGLUT1, with
VGLUT1 faster in hippocampal neurons, and VMAT2 faster in dopamine neurons.

Differences in recycling pool size might reflect expression of the two transporters at different synapses, or on different synaptic vesicles at the same synapse. In dopamine neurons, VMAT2 and VGLUT1 generally localize at the same boutons, but VMAT2 (and to a lesser extent VGLUT1) can appear alone even when the IRES construct ensures expression of both transporters, consistent with the observation of asymmetric synapses made by dopamine neurons in vivo (Hattori et al., 1991). However, VMAT2 and VGLUT1 colocalize entirely at the boutons of hippocampal neurons, where the difference in recycling pool size is even more substantial than in dopamine neurons, indicating that the differences in behavior must reflect expression on different pools of vesicles at the same bouton. Although controversial (Groemer and Klingauf, 2007), previous work has indeed suggested that different populations of synaptic vesicles mediate evoked and spontaneous neurotransmitter release (Sara et al., 2005; Fredj and Burrone, 2009). In addition to the functional differences, our work now demonstrates that recycling and reserve pool vesicles differ in the proportion of VMAT2 and VGLUT1. Higher expression of VMAT2 on reserve pool vesicles may help dopamine neurons to distinguish burst firing from pacemaking since, at least in Drosophila, reserve pool vesicles contribute to the release produced specifically by high-frequency stimulation (Kuromi and Kidokoro, 2000).

We also observed that, even in the absence of stimulation, $\mathrm{VMAT}^{+}{ }^{+}$membranes exhibit greater mobility along the axon than VGLUT1 ${ }^{+}$membranes, which remain almost entirely confined to synaptic boutons. With stimulation, GFP-VMAT2 fluorescence over boutons also declines to a greater extent than that of GFP-VGLUT1. This might reflect increased exocytosis of VMAT2, but a smaller proportion of VMAT2 resides in the recycling pool. Thus, the differences in dispersion probably reflect intrinsic differences in either diffusion along the plasma membrane or in endocytosis, which limits lateral spread. Indeed, differences in diffusion away from the active zone may contribute to the observed differences in endocytosis rate, particularly if the endocytosis machinery is concentrated at the release site (Roos et al., 2000; Marie et al., 2004). In addition, VMAT2 fails to recover at almost half of the boutons, and at those where recovery does occur, the rate of recovery is slower than for VGLUT1. This may again reflect differences in endocytosis, which is presumably required before vesicle reclustering, or alternatively, in the reclustering of newly formed synaptic vesicles at the release site. Differences in lateral diffusion, endocytosis, or reclustering may in turn account for the observed differences in targeting to recycling and reserve synaptic vesicle pools, and the subsequent behavior of VGLUT1 and VMAT2 on stimulation.

In summary, the expression of VMAT2 in dopamine neurons confers a number of properties that distinguish synaptic vesicles that release monoamines from those that release glutamate. During stimulation, midbrain dopamine neurons enable much more rapid retrieval of VMAT2 than hippocampal neurons. However, VMAT2 shows a number of surprising differences from VGLUT1 in both cell populations. After the stimulus, VMAT2 undergoes endocytosis more slowly than VGLUT1, and this may reflect the increased diffusion of VMAT2 along the axon before retrieval. The extent of exocytosis also differs between VMAT2 and VGLUT1, and this reflects reduced targeting of VMAT2 to the recycling pool, perhaps as a result of the endocytic pathway selected. These properties may in turn contribute to the responsiveness of dopamine release to burst stimulation that signals reward. 


\section{References}

Abeliovich A, Schmitz Y, Fariñas I, Choi-Lundberg D, Ho WH, Castillo PE, Shinsky N, Verdugo JM, Armanini M, Ryan A, Hynes M, Phillips H, Sulzer D, Rosenthal A (2000) Mice lacking alpha-synuclein display functional deficits in the nigrostriatal dopamine system. Neuron 25:239-252.

Balaji J, Ryan TA (2007) Single-vesicle imaging reveals that synaptic vesicle exocytosis and endocytosis are coupled by a single stochastic mode. Proc Natl Acad Sci U S A 104:20576-20581.

Benoit-Marand M, Jaber M, Gonon F (2000) Release and elimination of dopamine in vivo in mice lacking the dopamine transporter: functional consequences. Eur J Neurosci 12:2985-2992.

Benoit-Marand M, Borrelli E, Gonon F (2001) Inhibition of dopamine release via presynaptic $\mathrm{D} 2$ receptors: time course and functional characteristics in vivo. J Neurosci 21:9134-9141.

Chi P, Greengard P, Ryan TA (2001) Synapsin dispersion and reclustering during synaptic activity. Nat Neurosci 4:1187-1193.

Chuhma N, Zhang H, Masson J, Zhuang X, Sulzer D, Hen R, Rayport S (2004) Dopamine neurons mediate a fast excitatory signal via their glutamatergic synapses. J Neurosci 24:972-981.

Dal Bo G, St-Gelais F, Danik M, Williams S, Cotton M, Trudeau LE (2004) Dopamine neurons in culture express VGLUT2 explaining their capacity to release glutamate at synapses in addition to dopamine. J Neurochem 88:1398-1405.

Daniel JA, Galbraith S, Iacovitti L, Abdipranoto A, Vissel B (2009) Functional heterogeneity at dopamine release sites. J Neurosci 29:1467014680.

Dittman JS, Kreitzer AC, Regehr WG (2000) Interplay between facilitation, depression, and residual calcium at three presynaptic terminals. J Neurosci 20:1374-1385.

Dugast C, Brun P, Sotty F, Renaud B, Suaud-Chagny MF (1997) On the involvement of a tonic dopamine D2-autoinhibition in the regulation of pulse-to-pulse-evoked dopamine release in the rat striatum in vivo. Naunyn Schmiedebergs Arch Pharmacol 355:716-719.

Ferguson SM, Brasnjo G, Hayashi M, Wölfel M, Collesi C, Giovedi S, Raimondi A, Gong LW, Ariel P, Paradise S, O’Toole E, Flavell R, Cremona O, Miesenböck G, Ryan TA, De Camilli P (2007) A selective activitydependent requirement for dynamin 1 in synaptic vesicle endocytosis. Science 316:570-574.

Fernandez-Alfonso T, Ryan TA (2008) A heterogeneous "resting" pool of synaptic vesicles that is dynamically interchanged across boutons in mammalian CNS synapses. Brain Cell Biol 36:87-100.

Fernández-Alfonso T, Kwan R, Ryan TA (2006) Synaptic vesicles interchange their membrane proteins with a large surface reservoir during recycling. Neuron 51:179-186.

Finn JP 3rd, Edwards RH (1997) Individual residues contribute to multiple differences in ligand recognition between vesicular monoamine transporters 1 and 2. J Biol Chem 272:16301-16307.

Fortin DL, Nemani VM, Voglmaier SM, Anthony MD, Ryan TA, Edwards RH (2005) Neural activity controls the synaptic accumulation of $\alpha$-synuclein. J Neurosci 25:10913-10921.

Fredj NB, Burrone J (2009) A resting pool of vesicles is responsible for spontaneous vesicle fusion at the synapse. Nat Neurosci 12:751-758.

Fremeau RT Jr, Burman J, Qureshi T, Tran CH, Proctor J, Johnson J, Zhang H, Sulzer D, Copenhagen DR, Storm-Mathisen J, Reimer RJ, Chaudhry FA, Edwards RH (2002) The identification of vesicular glutamate transporter 3 suggests novel modes of signaling by glutamate. Proc Natl Acad Sci U S A 99:14488-14493.

Gandhi SP, Stevens CF (2003) Three modes of synaptic vesicular recycling revealed by single-vesicle imaging. Nature 423:607-613.

Garris PA, Ciolkowski EL, Pastore P, Wightman RM (1994) Efflux of dopamine from the synaptic cleft in the nucleus accumbens of the rat brain. J Neurosci 14:6084-6093.

Grace AA, Bunney BS (1984a) The control of firing pattern in nigral dopamine neurons: single spike firing. J Neurosci 4:2866-2876.

Grace AA, Bunney BS (1984b) The control of firing pattern in nigral dopamine neurons: burst firing. J Neurosci 4:2877-2890.

Granseth B, Odermatt B, Royle SJ, Lagnado L (2006) Clathrin-mediated endocytosis is the dominant mechanism of vesicle retrieval at hippocampal synapses. Neuron 51:773-786.

Gras C, Herzog E, Bellenchi GC, Bernard V, Ravassard P, Pohl M, Gasnier B, Giros B, El Mestikawy S (2002) A third vesicular glutamate transporter expressed by cholinergic and serotoninergic neurons. J Neurosci 22:5442-5451.

Groemer TW, Klingauf J (2007) Synaptic vesicles recycling spontaneously and during activity belong to the same vesicle pool. Nat Neurosci 10:145-147.

Hattori T, Takada M, Moriizumi T, Van der Kooy D (1991) Single dopaminergic nigrostriatal neurons form two chemically distinct synaptic types: possible transmitter segregation within neurons. J Comp Neurol 309: 391-401.

Hnasko TS, Chuhma N, Zhang H, Goh GY, Sulzer D, Palmiter RD, Rayport S, Edwards RH (2010) Vesicular glutamate transport promotes dopamine storage and glutamate corelease in vivo. Neuron 65:643-656.

Holt M, Cooke A, Wu MM, Lagnado L (2003) Bulk membrane retrieval in the synaptic terminal of retinal bipolar cells. J Neurosci 23:1329-1339.

Hooton JW (1991) Randomization tests: statistics for experimenters. Comput Methods Programs Biomed 35:43-51.

Jockusch WJ, Praefcke GJ, McMahon HT, Lagnado L (2005) Clathrindependent and clathrin-independent retrieval of synaptic vesicles in retinal bipolar cells. Neuron 46:869-878.

Johnson MD (1994) Synaptic glutamate release by postnatal rat serotonergic neurons in microculture. Neuron 12:433-442.

Kennedy RT, Jones SR, Wightman RM (1992) Dynamic observation of dopamine autoreceptor effects in rat striatal slices. J Neurochem 59:449455 .

Kuromi H, Kidokoro Y (2000) Tetanic stimulation recruits vesicles from reserve pool via a cAMP-mediated process in Drosophila synapses. Neuron 27:133-143.

Li H, Waites CL, Staal RG, Dobryy Y, Park J, Sulzer DL, Edwards RH (2005) Sorting of vesicular monoamine transporter 2 to the regulated secretory pathway confers the somatodendritic exocytosis of monoamines. Neuron 48:619-633.

Li Z, Murthy VN (2001) Visualizing postendocytic traffic of synaptic vesicles at hippocampal synapses. Neuron 31:593-605.

Mani M, Ryan TA (2009) Live imaging of synaptic vesicle release and retrieval in dopaminergic neurons. Front Neural Circuits 3:3.

Marie B, Sweeney ST, Poskanzer KE, Roos J, Kelly RB, Davis GW (2004) Dap160/intersectin scaffolds the periactive zone to achieve high-fidelity endocytosis and normal synaptic growth. Neuron 43:207-219.

Mendez JA, Bourque MJ, Dal Bo G, Bourdeau ML, Danik M, Williams S, Lacaille JC, Trudeau LE (2008) Developmental and target-dependent regulation of vesicular glutamate transporter expression by dopamine neurons. J Neurosci 28:6309-6318.

Mewhort DJ (2005) A comparison of the randomization test with the F test when error is skewed. Behav Res Methods 37:426-435.

Michael DJ, Wightman RM (1999) Electrochemical monitoring of biogenic amine neurotransmission in real time. J Pharm Biomed Anal 19:33-46.

Miesenböck G, De Angelis DA, Rothman JE (1998) Visualizing secretion and synaptic transmission with $\mathrm{pH}$-sensitive green fluorescent proteins. Nature 394:192-195.

Mitchell SJ, Ryan TA (2004) Syntaxin-1A is excluded from recycling synaptic vesicles at nerve terminals. J Neurosci 24:4884-4888.

Mosharov EV, Gong LW, Khanna B, Sulzer D, Lindau M (2003) Intracellular patch electrochemistry: regulation of cytosolic catecholamines in chromaffin cells. J Neurosci 23:5835-5845.

Pyle JL, Kavalali ET, Piedras-Rentería ES, Tsien RW (2000) Rapid reuse of readily releasable pool vesicles at hippocampal synapses. Neuron 28:221-231.

Rayport S, Sulzer D, Shi W-X, Sawasdikosol S, Monaco J, Batson D, Rajendran G (1992) Identified postnatal mesolimbic dopamine neurons in culture: morphology and electrophysiology. J Neurosci 12:4264-4280.

Rizzoli SO, Betz WJ (2005) Synaptic vesicle pools. Nat Rev Neurosci 6:57-69.

Roos J, Hummel T, Ng N, Klämbt C, Davis GW (2000) Drosophila Futsch regulates synaptic microtubule organization and is necessary for synaptic growth. Neuron 26:371-382.

Sankaranarayanan S, Ryan TA (2000) Real-time measurements of vesicleSNARE recycling in synapses of the central nervous system. Nat Cell Biol 2:197-204.

Sankaranarayanan S, Ryan TA (2001) Calcium accelerates endocytosis of vSNAREs at hippocampal synapses. Nat Neurosci 4:129-136.

Sankaranarayanan S, De Angelis D, Rothman JE, Ryan TA (2000) The use of pHluorins for optical measurements of presynaptic activity. Biophys J 79:2199-2208. 
Sara Y, Virmani T, Deák F, Liu X, Kavalali ET (2005) An isolated pool of vesicles recycles at rest and drives spontaneous neurotransmission. Neuron 45:563-573.

Schäfer MK, Varoqui H, Defamie N, Weihe E, Erickson JD (2002) Molecular cloning and functional identification of mouse vesicular glutamate transporter 3 and its expression in subsets of novel excitatory neurons. J Biol Chem 277:50734-50748.

Schultz W (2002) Getting formal with dopamine and reward. Neuron 36:241-263.

Staal RG, Mosharov EV, Sulzer D (2004) Dopamine neurons release transmitter via a flickering fusion pore. Nat Neurosci 7:341-346.

St-Gelais F, Legault M, Bourque MJ, Rompré PP, Trudeau LE (2004) Role of calcium in neurotensin-evoked enhancement in firing in mesencephalic dopamine neurons. J Neurosci 24:2566-2574.

Sulzer D, Joyce MP, Lin L, Geldwert D, Haber SN, Hattori T, Rayport S (1998) Dopamine neurons make glutamatergic synapses in vitro. J Neurosci 18:4588-4602.

Sun JY, Wu XS, Wu LG (2002) Single and multiple vesicle fusion induce different rates of endocytosis at a central synapse. Nature 417:555-559.
Tan PK, Waites C, Liu Y, Krantz DE, Edwards RH (1998) A leucine-based motif mediates the endocytosis of vesicular monoamine and acetylcholine transporters. J Biol Chem 273:17351-17360.

Varga V, Losonczy A, Zemelman BV, Borhegyi Z, Nyiri G, Domonkos A, Hangya B, Holderith N, Magee JC, Freund TF (2009) Fast synaptic subcortical control of hippocampal circuits. Science 326:449-453.

Voglmaier SM, Kam K, Yang H, Fortin DL, Hua Z, Nicoll RA, Edwards RH (2006) Distinct endocytic pathways control the rate and extent of synaptic vesicle protein recycling. Neuron 51:71-84.

Wise RA (2004) Dopamine, learning and motivation. Nat Rev Neurosci 5:483-494.

Xu J, McNeil B, Wu W, Nees D, Bai L, Wu LG (2008) GTP-independent rapid and slow endocytosis at a central synapse. Nat Neurosci 11:45-53.

Yamaguchi T, Sheen W, Morales M (2007) Glutamatergic neurons are present in the rat ventral tegmental area. Eur J Neurosci 25:106-118.

Zhang Q, Li Y, Tsien RW (2009) The dynamic control of kiss-and-run and vesicular reuse probed with single nanoparticles. Science 323: $1448-1453$. 